\title{
MODELIMG THE TOTAL ENERGY COSTS OF RESISTAMCE EXERCISE: A WORK IN PROGRESS
}

\author{
Christopher B. Scott, ${ }^{1, A}$, Dictor M. Reis ${ }^{2, D}$ \\ 1 University of Southern Maine, Exercise, Health and Sport Sciences, USA \\ ${ }^{2}$ Research Centre for Sports Sciences, Health Sciences \& Human Development, University of Trás-os-Montes \& Alto Douro (UTAD), Portugal

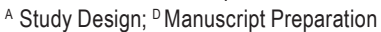

\author{
Address for corpespondence: \\ Christopher B. Scott, PhD \\ University of Southern Maine, Exercise, Health and Sport Sciences \\ Gorham, ME 04038, USA \\ E-mail: cscott@usm.maine.edu
}

\begin{abstract}
Ahstract. We present an aerobic and anaerobic, exercise and recovery energy cost model of intermittent energy costs utilizing task (work, Joules) as opposed to rate (per minute) measurements. Low to moderate intensity steady state exercise energy costs are typically portrayed as the volumetric rate at which oxygen is consumed $\left(\mathrm{VO}_{2} \mathrm{~L} \mathrm{~min}{ }^{-1}\right)$, where a proportionate upward climbing linear relationship is profiled with an increasing power output; add to this the concept of the anaerobic threshold and energy costs increase with more intense aerobic exercise in disproportion to $\mathrm{VO}_{2} \mathrm{~L} \mathrm{~min}^{-1}$ measurements. As a per task function, intermittent work and recovery bouts contain a combined estimate of total costs, that is as $\mathrm{kJ}$ or kcal (not $\mathrm{kJ} \cdot \mathrm{min}^{-1}$ or $\left.\mathrm{kcal} \cdot \mathrm{min}^{-1}\right)$. Adopting this approach to describe single and multiple sets of resistance training, the model that emerges for intermittent resistance exercise portrays linearity between equivalent work and total energy costs that differs proportionately among conditions - "continuous" muscular endurance vs. intermittent higher load strength work, moderately paced vs. slower and faster conditions, smaller vs. larger working muscle masses and failure (fatigue) vs. non-failure states. Moreover, per kcal (or kJ) of total energy costs, work ( $\mathrm{J}$ ) is more inefficient with a greater load and lower repetition number as opposed to lower resistance with an increased number of repetitions. The concept of energy costs rising disproportionately with increased or prolonged work does not appear to apply to resistance exercise.
\end{abstract}

Key world: intermittent exercise, weight lifting, oxygen uptake

\section{Cost per task (not per minute)}

Validation has not been provided for the almost universal use of steady state per minute exercise oxygen uptake measurements (e.g., $\mathrm{VO}_{2} l \cdot \mathrm{min}^{-1}$ ) in the estimation of the energy costs of non-steady state exercise (Scott, Reis, 2014). An example of this practice suggests that a modified per minute oxygen uptake measure taken exclusively in recovery may better estimate resistance exercise energy costs (Vezina, Ananian, Campbell, Meckes, Ainsworth, 2014). Again, there appears to be no precedent in eliminating exercise energy costs when estimating overall exercise and recovery energy costs. Indeed, this practice instead reveals the limitations of steady state per 
minute measurements. We promote an estimate of both the exercise and recovery energy costs of intermittent exercise along with their aerobic and anaerobic metabolic components as obligatory to the overall total energy cost - resistance exercise and recovery costs should be summed not averaged.

We have adopted a per task work model that allows for the collection of all energy cost components - aerobic and anaerobic, exercise and recovery - as $\mathrm{kJ}$ or $\mathrm{kcal}$ (not $\mathrm{kJ} \cdot \mathrm{min}^{-1}$ or $\mathrm{kcal} \cdot \mathrm{min}^{-1}$ ) for resistance exercise. Intermittent resistance work $(\mathrm{J})$ was electronically recorded as the product of vertical bar movement (on a Smith machine) and weight lifted (with a coefficient of variation of $0.25 \%$ between reps and $0.75 \%$ between sets) (inertia was not accounted for) (Scott, Croteau, Ravlo, 2009).

Contrary to the concept of an anaerobic threshold as applied to higher intensity aerobic-type exercise, our model has yet to reveal any disproportionate increase in energy costs at higher work volumes (Scott, Reis, 2014). Total energy costs instead rise linearly with work and as the conditions of lifting change - with a greater number of higher load intermittent sets, with slower and faster lifting speeds, with the use of larger muscle masses and when lifting to fatigue - these costs increase in linear proportion to work (Figure 1).

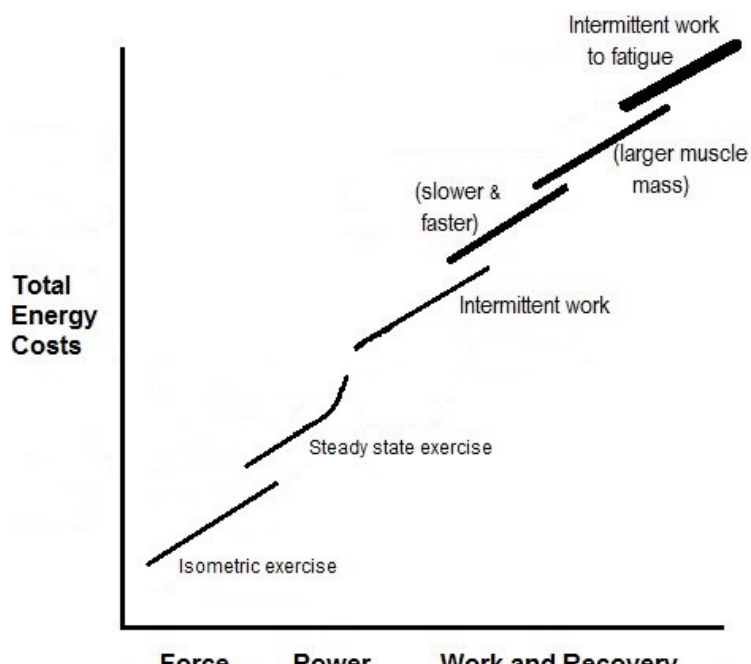

Figune 1 The total energy costs (aerobic and anaerobic, exercise and recovery) of force, power, and work bouts are hypothetically depicted. Isometric exercise reveals the lowest energy cost. With steady state power, energy costs increase disproportionately after the anaerobic threshold is reached. Work and recovery are depicted as rising in linear and proportional fashion under a variety of conditions: with greater load intermittent bouts, with slower and faster lifting speeds, and with the use of an increased muscle mass; each has a greater total cost than equivalent work performed at a moderate pace (parentheses indicate the interchangeability of conditions). Lifting to muscular failure increases total energy costs further, likewise in a proportional linear manner as compared to non-failure (not an exact scale, increased variability is denoted via increased line thickness).

\section{Movement}

Comparison of three Tabata-type calisthenic squat routines revealed that isometric exercise conditions provide the lowest total energy costs as compared to isotonic and plyometric conditions (Scott, Nelso, Martin, Ligotti, 2015). 
Based on force-velocity relationships however, the greatest force skeletal muscle can apply is under isometric conditions. From such perspectives isometric exercises represent an ideal means of strength and conditioning training, but they do not maximize energy costs. Physical movement appears integral to maximizing the caloric costs of exercise, and that likely includes resistance exercise.

\section{Intermittent Conditions}

Using thermodynamic considerations regarding metabolism as opposed to combustion, the oxidation of glucose has been suggested at $5.0 \mathrm{kcal}(21.1 \mathrm{~kJ})$ per liter of oxygen consumed; with fat and lactate as fuels the conversion in terms of one liter of oxygen consumed is $4.7 \mathrm{kcal}$ (19.6 kJ) (Scott, 2014). Put another way, for every $\mathrm{kcal}$ of energy cost, 0.20 liters of oxygen are consumed when using glucose as a fuel $\left(1 \mathrm{kcal}=0.20 \mathrm{LO}_{2}\right)$; with fat and lactate oxidation, for every $1 \mathrm{kcal}$ of energy cost, 0.21 liters of oxygen are consumed $\left(1 \mathrm{kcal}=0.21 \mathrm{LO}_{2}\right)$. Thus, if recovery costs $20 \mathrm{kcal}(84 \mathrm{~kJ})$ then with glucose as a fuel, 4.0 liters of oxygen are consumed; with fat and lactate oxidation, 4.3 liters of oxygen are consumed.

Because fuel selection is dependent on exercise "intensity" it appears likely that higher intensity intermittent exercise periods would consist primarily of glucose oxidation (as well as use of the high energy phosphates - ATP and $\mathrm{PCr}$ ). During intermittent recovery periods, when muscle is not contracting (i.e., zero "intensity"), lactate and fats are the likely predominate fuel source. We view 2 sets or more of resistance exercise as having 2 or more respective recovery periods that are all summed to provide a single recovery energy cost estimate; the term 'excess post-exercise oxygen consumption' (EPOC) identifies the last recovery period by name only (Scott, 2012a).

In reference to substrate utilization, a larger quantitative (proportional) difference would exist in the oxygenrelated costs between steady state exercise (power output) consisting of a single recovery period and equivalent work performed under intermittent conditions (Figure 1). A qualitative difference is further apparent between low to moderate intensity steady state and higher intensity intermittent conditions, with the potential for the latter to exhibit greater body composition changes (fat loss) (Boutcher, 2011; Hunter, Weinsier, Bamman, Larson, 1998).

\section{Total energy cost components}

Resistance and aerobic exercise are different and highly specific in terms of physiological responses (Knuttgen, 2007). Indeed, under typical resistance training loads, working skeletal muscle does not appear to receive a steady supply of oxygenated blood (Tamaki, Uchiyama, Tamura, Nakano, 1994). In fact for intermittent non-steady state weight lifting, the aerobic-anaerobic exercise-recovery energy cost of work per set is quite opposite that of the steady state model: 1) the volume of oxygen consumed during the actual weight lifting (exercise) period $\left(\mathrm{L} \mathrm{O}_{2}\right)$ represents the lowest contribution to overall energy costs, 2) recovery oxygen uptake $\left(\mathrm{L}_{2}\right)$ often represents the largest energy cost contribution (representing, in part, the so-called alactic component of ATP and phosphocreatine (PC) utilization and re-synthesis) along with, 3) anaerobic (glycolytic) costs that are often quite extensive as portrayed by blood lactate levels that rise predictably with work (Scott, Croteau, Ravlo, 2009; Scott, Leighton, Ahearn, McManus, 2011; Scott, 2012a). With low to moderate intensity steady rate power output: 1) as a per minute function, exercise oxygen uptake $\left(\mathrm{VO}_{2} \mathrm{l} \cdot \mathrm{min}^{-1}\right)$ always represents the largest contributor to overall costs, 2) oxygen uptake in recovery (EPOC) may or may not contribute significantly to overall costs and, 3) anaerobic costs need not be considered.

Unfortunately, at this point in time, no universal consensus exists regarding how anaerobic (glycolytic) energy costs should be quantified. From such a vantage point and the possibility that glycolytic costs may not contribute 
significantly to overall energy costs, it appears likely that the energy expenditure focus for resistance exercise will continue with oxygen-only measurements. Recent evidence however suggests that blood lactate measurements can in fact provide a reasonable marker of an anaerobic glycolytic energy cost component, that is under the conditions of work and not power output (Buitrago, Wirtz, Flenker, Kleinoder, 2014; Gorostiaga et al. 2014).

\section{Inefficiency}

Further information is found when examining the ratio of the amount of work performed along with the associated aerobic and anaerobic, exercise and recovery costs in an attempt to recognize the work-to-cost ratio in regard to the overall work performed. For single sets of submaximal lifts, where muscular failure or fatigue was not reached, it is apparent that the work-to-cost ratio reaches a plateau with increasing repetitions (Figure 2). A similar relationship also was found when lifting to muscular failure (Figure 3). The anaerobic threshold concept of disproportionately increasing energy costs is not portrayed by our cost per task analysis. Indeed the opposite appears, efficiency improves as more work is completed. This unique relationship appears to be the result of an increasing aerobic and anaerobic exercise energy cost component with work, yet with recovery and/or EPOC costs, linearity with work is not straight-forward (Scott, Croteau, Ravlo, 2009; Scott, Leighton, Ahearn, McManus, 2011; Scott, Leary, TenBraak, 2011).

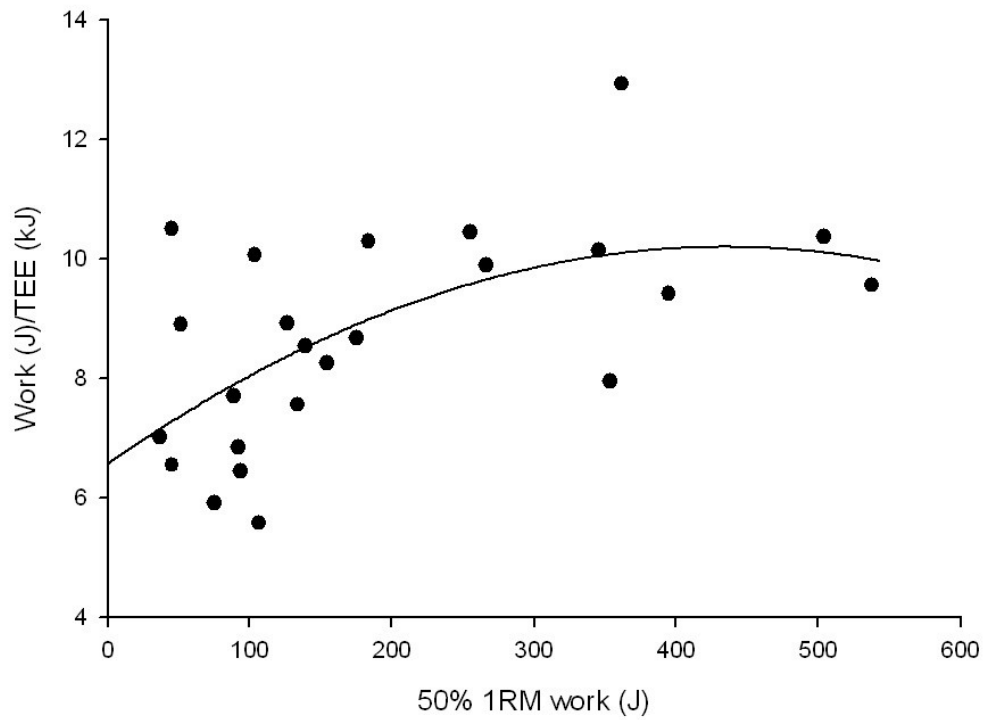

Figure 2. The work $(J)$ to total energy costs $(k J)$ ratio is shown for submaximal lifting (50\% 1-RM) of 1-set of the bench press exercise, muscular failure (or fatigue) was not an endpoint (Scott, Croteau, Ravlo, 2009). The data points represent 7, 14 and 21 repetitions. A quadratic equation was used to provide a line of best fit (SigmaPlot v.12). Efficiency appears to improve as the amount of work increases; as part of the sum of aerobic and anaerobic energy costs, EPOC appears to plateau as resistance work increases. 


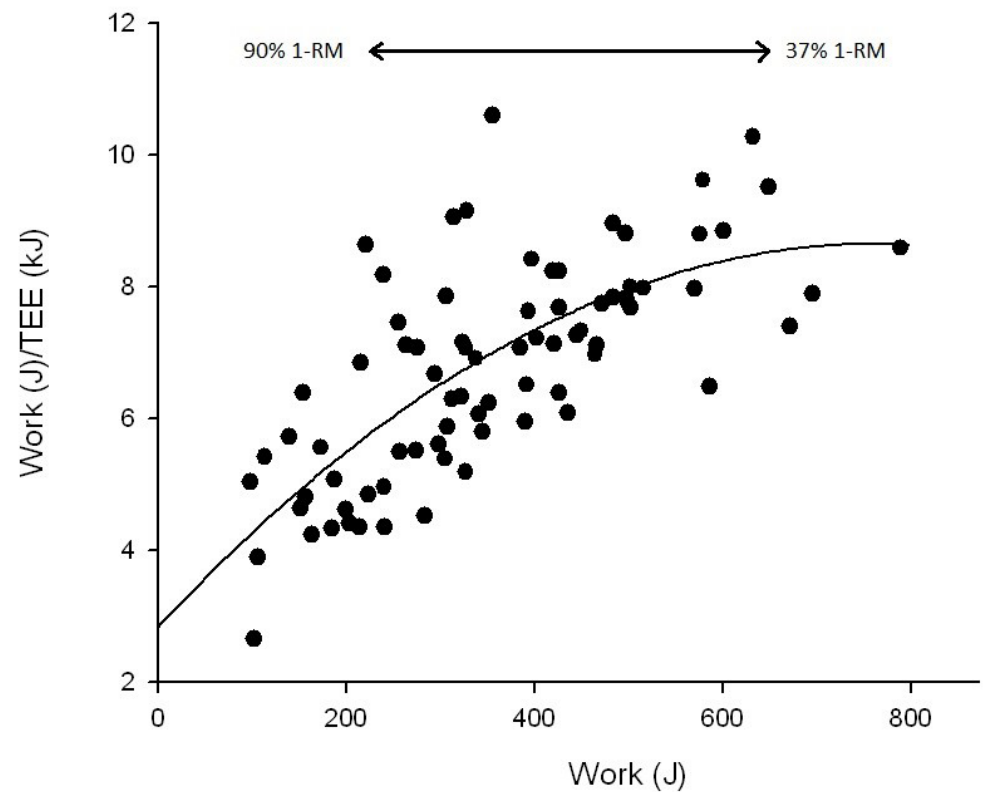

Figule 3. The work (J) to total energy expenditure (kJ) ratio is shown for 1-set of bench press lifting to muscular failure at: $90 \%, 80 \%$, $70 \%, 56 \%, 46 \%$ and $37 \%$ of a one repetition maximum load (1-RM) (Scott, Leighton, Ahearn, McManus, 2011). A quadratic equation was used to provide the line of best fit (SigmaPlot v.12). At the lowest load (37\% 1-RM), as with submaximal lifts (Figure 2), greater work is completed in relation to a lower total energy cost - efficiency appears to improve. An improving efficiency with increased work appears to be the result of an EPOC (and/or recovery) volume that plateaus as work increases (for single sets and double sets, respectively) (Scott, Leary, TenBraak, 2011).

When single lifts were performed to failure it was the largest load - at $90 \%$ of a 1-RM - that had the least amount of work completed per kcal of energy cost: 1 kcal per 21 Joules of work (Table 1; Figure 3). Compare this to the muscular endurance-type workload (37\% 1-RM) where for every kcal utilized, 34 Joules of work were completed. The same pattern was seen when lifting to failure with consecutive sets (Scott, Leighton, Ahearn, McManus, 2011). The concept of an improved efficiency may represent a real difference between intermittent work bouts and steady state power output (see Woledge, 1998).

Table 1. Inefficiency of the Bench Press: 1 set to failure

\begin{tabular}{ccccc}
\hline \% 1-RM & Reps & Total Cost (kcal) & Work $(\mathrm{J})$ & Joules per kcal $\left(\mathrm{Jkcal}^{-1}\right)$ \\
\hline 37 & 36 & 15 & 514 & 34 \\
46 & 25 & 14 & 439 & 31 \\
56 & 20 & 14 & 426 & 30 \\
70 & 12 & 12 & 342 & 28 \\
80 & 8 & 11 & 274 & 25 \\
90 & 4 & 8 & 167 & 21 \\
\hline
\end{tabular}

Source: Scott, Leighton, Ahearn, McManus (2011). 
Greater work costs proportionately more as compared to less, regardless of the load (Figure 1). However, utilizing the concept of inefficiency (Table 1), the hypothetical completion of 3 sets at a high load of $90 \%$ of a one repetition maximum (1-RM) - 12 total repetitions (501 J) at a cost of $24 \mathrm{kcal}$ - would be required to closely match the work of 1 set at 37\% 1-RM (low load) consisting of 36 repetitions (514 J), with a 9 kcal increase in total costs for the former. Per volume of work $(\mathrm{J})$, the use of a higher load in association with a greater number of intermittent exercise and recovery periods is suggested to increase overall or total energy costs.

\section{Muscle mass and work}

Several single-set resistance exercises were compared in terms of the overall work completed and the associated total energy costs (Scott, Luchini, Knausenberger, Steitz, 2014). It needs to be kept in mind that the load and repetition combinations for each lift varied tremendously; there also may be a gender effect as well. Even so, at equivalent submaximal work $(\mathrm{J})$, the total energy cost was lowest for the flat and incline chest press (smallest muscle mass), larger for the squat (moderate muscle mass) and greatest for the deadlift (largest muscle mass); total energy costs rose linearly with work for each lift.

While certainly requiring more research, we currently portray the energy costs for the completion of a given amount of work to be proportionately larger the greater the amount of recruited muscle mass (Figure 1).

\section{Lifting speed}

The effects of lifting speed on total energy costs also has undergone partial examination. Utilizing the bench press exercise at 70\% 1-RM where 3 sets of 5 repetitions were completed (15 overall reps) at two different lifting speeds - slow ( 5 seconds per rep) and moderate ( 3 seconds per rep) - two similar workloads were compared (Scott, 2012b). With the lifting speed slowed down from 15 seconds per set to 25 seconds per set, total costs increased by $6 \mathrm{kcal}$ - inefficiency drops from 33 to 23 Joules of work per kcal.

We have not yet completed an energy cost and work study at rapid lifting speeds but, at faster lifting speeds inertia almost certainly plays a greater role and overall costs per task should increase as compared to moderate lifting speeds. Estimating work as the product of load, repetition, set number, and time to complete repetitions, it has been shown that explosive muscular contractions can indeed increase the energy costs of resistance exercise (Mazzetti, Douglass, Yocum, Harber, 2007).

\section{Lifting to failupe}

Based on a retrospective investigation, lifting a weight to failure for one set promotes the largest total energy cost, also rising linearly and in parallel proportion as compared with submaximal equivalent work (Figure 1) (Scott, Earnest, 2011). Again, our model does not indicate the presence of an anaerobic threshold description for resistance exercise, when "extra" aerobic costs continue to rise disproportionately. With weight lifting (as opposed to steady state exercise), where blood flow to muscle is likely occluded or arrested, the "extra" total costs promoted by muscular failure appear fixed, regardless of the amount of work completed. However, at the lowest loads and as more work is completed, efficiency appears to improve, both with and without muscle failure (Figures 2 and 3 ). 


\section{Limitations}

Perhaps the greatest limitation to the study of resistance exercise is in the quantification of work. On the one hand tradition dictates some compendium of a percentage of a repetition maximum (\% RM), weight lifted, repetition number and sets completed. On the other hand the unit of measurement for work is designated as the Joule - a product of force, distance and inertia. While power output (Watts) is well understood, Joules of work is a foreign language to many involved with resistance training, including exercise scientists, though it has been shown to be the most valid method of quantifying resistance training "volume" (McBride et al., 2009). The concept of EPOC versus recovery oxygen uptake also presents as a limitation. Four sets of a specific resistance exercise for example, would consist of 3 recovery periods and 1 EPOC. Such representation is suggestive of recovery being separate from EPOC when in fact they both represent non-exercise phases (i.e., 4 overall recovery periods with EPOC representing the last and longest of those periods). The total exercise energy cost model presented has been designed to examine resistant exercise 1 set at a time and may not be descriptive of a complete resistance training workout.

\section{Practical implications and conclusions}

Our per task Work model $(\mathrm{J})$ represented by a total energy cost estimate $(\mathrm{kJ})$ - aerobic and anaerobic, exercise and recovery - provides an alternative method of analysis as compared to traditional steady state per minute measures. At present, for intermittent resistance exercise with independent recovery periods, we propose linear and proportional increases with total energy costs and work. Moreover, per kcal of total energy costs, work is most inefficient with a greater load and lower repetition number as opposed to lower resistance with an increased number of repetitions.

The energy cost - work relationships presently depicted serve as a blueprint or framework for the design of exercise-related weight loss programs where the absolute numerical cost of the exercise may not be known, but the relative description of the exercise format can suggest lower and higher total energy cost profiles. Brief, intermittent, high-load, resistance exercises with recovery periods, utilizing large muscle masses, performed slowly or quickly, to failure, would hypothetically promote the greatest total energy cost for a given volume of work.

\section{Acknowledgement}

We wish to acknowledge Prof. Pedro J. Benito of the LFE Research Group, Department of Health and Human Performance, Faculty of Physical Activity and Sport Science-INEF, Technical University of Madrid, Spain, for allowing some of this material to be presented at the International Congress of Overweight and Obesity Treatment December 2011 and the 7th International Symposium in Strength Training, December 2014.

This information also was presented in part at the $3^{\text {rd }}$ International Symposium on Strength \& Conditioning, hosted July 2013 at the University of Trás-os-Montes \& Alto Douro, Portugal. 


\section{References}

Boutcher, S.H. (2011). High-intensity intermittent exercise and fat loss. J Obesity. (Article ID 868305). DOI: 10.1155/2011/868305.

Buitrago, S., Wirtz, N., Flenker, U., Kleinoder, H. (2014). Physiological and metabolic responses as function of the mechanical load in resistance exercise. Appl Phys Nutr Met, 39, 345-350. DOI: 10.1139/apnm-2013-0214.

Gorostiaga, E.M., Navarro-Amezqueta, I., Calbert, J.A.L., Sanchez-Medina, L., Cusso, R., Granados, C., Izquierdo, M. (2014). Blood ammonia and lactate as markers of muscle metabolites during leg press exercise. J Strength Cond Res, 28, 2775-2785. DOI: 10.1519/JSC.0000000000000496.

Hunter, G.R., Weinsier, R.L., Bamman, M.M., Larson, D.E. (1998). A role for high intensity exercise on energy balance and weight control. Int J Obesity, 22, 489-493.

Knuttgen, H.G. (2007). Strength training and aerobic exercise: comparison and contrast. J Strength Cond Res, 21, 973-978. DOI: 10.1519/R-505011.1.

Mazzetti, S., Douglass, M., Yocum, A., Harber, M. (2007). Effect of explosive versus slow contractions and exercise intensity on energy expenditure. Med Sci Sports Exer, 39, 1291-1301.

McBride, J.M., McCaulley, G.O., Cormie, P., Nuzzo, J.L. Cavill, M.J., Triplett, N.T. (2009). Comparison of methods to quantify volume during resistance exercise. J Strength Cond Res, 23, 106-110.

Scott, C.B., Nelson, E., Martin, S., Ligotti, B. (2015). Total energy costs of 3 Tabata-type calisthenic squatting routines: isometric, isotonic and jump. Eur J Human Movement, 8, 53-59.

Scott, C.B., Reis, V. (2014). Steady state models provide an invalid estimate of intermittent resistance-exercise energy costs. Eur J Human Movement, 33, 70-78.

Scott, C.B., Luchini, A., Knausenberger, A., Steitz, A. (2014). Total energy costs - aerobic and anaerobic, exercise and recovery - of five resistance exercises. Cent Euro J Sport Sci Med, 8, 53-59.

Scott, C.B. (2014). Combustion, respiration and intermittent exercise: a theoretical perspective on oxygen uptake and energy expenditure. Biology, 3, 255-263. DOI: 10.3390/biology3020255.

Scott, C.B. (2012a). Oxygen costs peak after resistance exercise sets: a rationale for the importance of recovery over exercise. J Ex Phys, 15, 1-7.

Scott, C.B. (2012b). The effect of time-under-tension and weight lifting cadence on aerobic, anaerobic, and recovery energy expenditures: 3 submaximal sets. Appl Phys Nutr Met, 37, 252-256. DOI: 10.1139/h11-158.

Scott, C.B., Earnest, C.P. (2011). Resistance exercise energy expenditure is greater with fatigue as compared to non-fatigue. J Ex Phys, $14,1-10$.

Scott, C.B. Leighton, B.H., Ahearn, K.J., McManus, J.J. (2011). Aerobic, anaerobic and excess postexercise oxygen consumption energy expenditure of muscular endurance and strength: 1-set of bench press to muscular failure. J Strength Cond Res, 25, 903-908. DOI: 10.1519/JSC.0b013e3181c6a128.

Scott, C.B., Leary, M.P., TenBraak, A.J. (2011). Energy expenditure characteristics of weight lifting: 2 sets to fatigue. Appl Phys Nutr Met, 36, 115-120. DOI: 10.1139/H10-093.

Scott, C.B., Croteau, A., Ravlo, T. (2009). Energy expenditure before during and after the bench press. J Strength Cond Res, 23 , 611-618. DOI: 10.1519/JSC.0b013e31818c2845.

Tamaki, T., Uchiyama, S., Tamura, T., Nakano, S. (1994). Changes in muscle oxygenation during weight-lifting exercise. Eur J Appl Phys, 68, 465-469.

Vezina, J.W., Ananian, C.A.D., Campbell, K.D., Meckes, N., Ainsworth, B.E. (2014). An examination of the differences between two methods of estimating energy expenditure in resistance training activities. J Strength Cond Res, 28, 1026-1031. DOI: 10.1519/ JSC.0000000000000375.

Woledge, R.C. (1998). Possible effects of fatigue on muscle efficiency. Acta Phys Scand, 162, 267-273.

Cite this anticle aS: Scott, C.B., Reis, V.M. (2016). Modeling the Total Energy Costs of Resistance Exercise: a Work in Progress. Central

European Journal of Sport Sciences and Medicine, 14 (2), 5-12. DOI: 10.18276/cej.2016.2-01. 